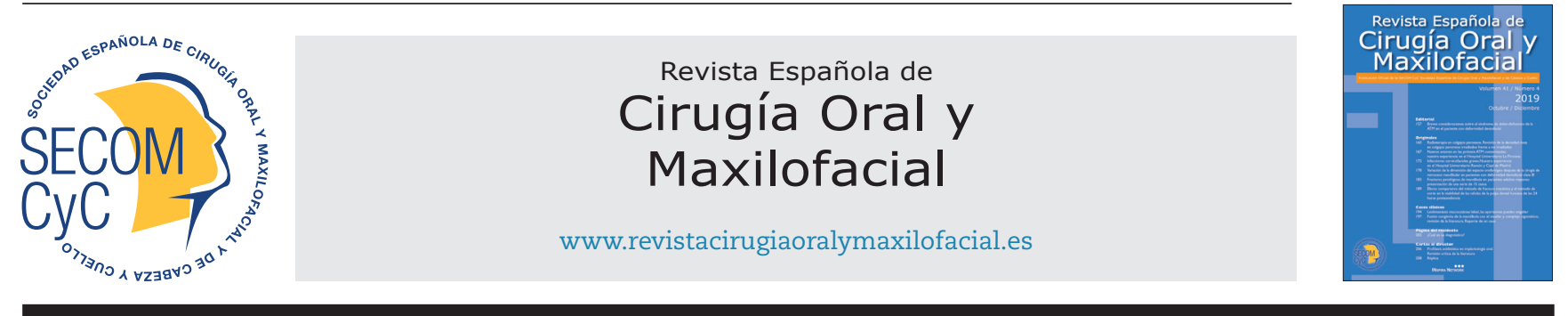

\title{
Original
}

\section{Infecciones cervicofaciales graves. Nuestra experiencia en el Hospital Universitario Ramón y Cajal de Madrid}

\author{
Álvaro Ranz Colio*, Elena Baranda Manterola, Álvaro Pastor Garrido, Ángela Bueno de \\ Vicente, Fernando Almeida Parra y Julio Acero Sanz
}

Servicio de Cirugía Oral y Maxilofacial. Hospital Universitario Ramón y Cajal, Madrid, España

INFORMACIÓN DEL ARTÍCULO

Historia del artículo:

Recibido el 11 de mayo de 2019

Aceptado el 12 de septiembre de 2019

Palabras clave:

Infección odontogénica, sensibilidad antibiótica, espacios cervicales, infección cervical grave.

\section{R E S U M E N}

Introducción: Las infecciones cervicofaciales constituyen un motivo de consulta muy frecuente en los servicios de Urgencias de nuestro país, siendo con frecuencia causa de gran morbilidad y de importantes complicaciones, incluyendo el compromiso de la vía aérea. Por todo ello, un diagnóstico y tratamiento precoces son de vital importancia.

El objetivo principal es realizar un estudio observacional retrospectivo sobre los pacientes diagnosticados de infección cervicofacial grave en nuestro centro, analizando múltiples variables demográficas, el tratamiento administrado, la duración del ingreso y las complicaciones observadas.

Material y métodos: Estudio observacional descriptivo retrospectivo sobre una muestra de 47 pacientes diagnosticados de infección cervicofacial grave en nuestro centro entre abril de 2016 y marzo de 2018. Se recogen y analizan múltiples variables: sexo, etiología, clínica asociada, aislamiento microbiológico, tratamiento, comorbilidades, duración de ingreso y complicaciones asociadas.

Resultados: El 51 \% de la muestra fueron pacientes menores de 50 años sin comorbilidades asociadas. Las comorbilidades más frecuentes fueron los hábitos tóxicos (tabaco y alcohol), hipertensión arterial y diabetes. Respecto a la etiología, el 91 \% fueron odontogénicas, siendo los cordales inferiores las piezas dentales afectadas con mayor frecuencia (79,06 \%). La clínica característica de presentación fue la tríada de tumefacción facial, dolor y trismus (hasta el $60 \%$ ). El espacio cervicofacial afectado con mayor frecuencia fue el submandibular (56\%). El aislamiento microbiológico mostró que la mayoría fueron infecciones polimicrobianas mixtas (18 de 38 aislamientos) con predominio de los grupos Streptococo y Prevotella.

*Autor para correspondencia:

Correo electrónico: alvaro.ranzcolio@gmail.com (Álvaro Ranz Colio).

DOI: 10.20986/recom.2019.1068/2019

1130-0558/@ 2019 SECOM. Publicado por Inspira Network. Este es un artículo Open Access bajo la licencia CC BY-NC-ND (http:// creativecommons.org/licenses/by-nc-nd/4.0/). 
Las complicaciones encontradas fueron: dos pacientes con obstrucción de vía aérea superior que precisaron traqueostomía previa intubación, un hematoma postquirúrgico, tres reintervenciones por mala evolución clínica y un paciente con mediastinitis.

Conclusiones: De los resultados obtenidos podemos concluir que la etiología odontogénica es la más frecuente, siendo el espacio submandibular el más afectado. En el Hospital Ramón y Cajal de Madrid, la mayoría de las infecciones cervicofaciales graves son mixtas con microrganismos aislados aerobios y anaerobios. El tratamiento combinado con cirugía y antibioterapia intravenosa fue de elección. Amoxicilina-Clavulánico fue el antibiótico más utilizado. Las complicaciones evolutivas graves son poco frecuentes con un tratamiento adecuado.

\section{Severe cervicofacial infections. Our experience in Ramón y Cajal University Hospital, Madrid}

\section{A B S T R A C T}

\section{Keywords:}

Odontogenic infections, antibiotic sensitivity, cervical compartiments, severe cervical infection.
Introduction: Cervical infections are a very common reason for consultation in the emergency services of our country. However, in certain cases, these infections are a cause of a great morbidity and important complications, including the compromise of the upper airway. For all these reasons, early diagnosis and treatment are of a great importance.

The main objective is to perform a retrospective study of patients diagnosed with severe cervicofacial infection in our department, analyzing multiple demographic variables, treatment administered, time of hospitalization and complications observed.

Material and methods: Retrospective descriptive observational study based on a sample of 47 patients diagnosed with severe cervicofacial infection in our center between April 2016 and March 2018. Multiple variables were collected, among which are: sex, etiology, associated symptoms, microbiological isolation, treatment established, comorbidities, time of hospital admission and complications.

Results: $51 \%$ of the sample were patients under 50 years of age without comorbidities. The most frequent comorbidities were toxic habits (tobacco and alcohol), hypertension and diabetes. Regarding the etiology, $91 \%$ were odontogenic, being the third inferior molars the most frequently affected $(79.06 \%)$. The characteristic clinical presentation was the triad of facial swelling, pain and trismus (up to $60 \%$ ). The most frequently affected cervicofacial space was the submandibular space (56\%).

The microbiological isolation showed that the majority were mixed polymicrobial infections (18 of 38 isolates) with predominance of the Streptococcus and Prevotella groups.

The complications that we found were: two upper airway obstructions, a cervical bleeding, three reinterventions for a bad clinical evolution and a patient with mediastinitis.

Conclusions: We can conclude that odontogenic etiology is the most frequent in severe cervicofacial infections, with the submandibular space being the most affected. Most of severe cervicofacial infections in Ramón y Cajal Hospital were polymicrobial and mixed infections. The combined treatment with surgery and intravenous antibiotic therapy was the therapeutic option chosen for all patients. Amoxicillin-Clavulanic was the most used broad-spectrum antibiotic. Complications are uncommon with an adequate treatment.

\section{INTRODUCCIÓN}

Las infecciones cervicofaciales son un motivo de consulta frecuente dentro de las urgencias hospitalarias y pueden ser motivo de importante morbilidad y de graves complicaciones, entre otras el compromiso de la vía aérea ${ }^{1,2}$.

La etiología más frecuente es la odontogénica; otras causas posibles, pero menos prevalentes, son la patología de glándulas salivales, las amígdalas faríngeas o las infecciones cutáneas 3 .

Las infecciones cervicofaciales de origen odontogénico desde el punto de vista microbiológico son infecciones mixtas con microorganismos aerobios y anaerobios de la cavidad oral ${ }^{4,5}$.

El abordaje terapéutico suele ser médico-quirúrgico. La cirugía puede indicarse para el tratamiento etiológico de la in- fección y el drenaje de material purulento, así como para la exploración de espacios tisulares profundos ${ }^{3}$.

El tratamiento médico consiste en antibioterapia, medidas de soporte hidroelectrolítico y corticoterapia.

El objetivo de este estudio es analizar múltiples variables epidemiológicas de los pacientes con infecciones cervicofaciales graves manejados en nuestro centro, revisar el manejo terapéutico y las complicaciones evolutivas observadas durante el ingreso.

\section{MATERIAL Y MÉTODOS}

El presente estudio muestra un análisis observacional y retrospectivo de una serie de 47 casos de infecciones cervicofa- 
ciales graves, diagnosticados y manejados en el Servicio de Cirugía Oral y Maxilofacial del Hospital Ramón y Cajal, de Madrid, España, entre los meses de abril de 2016 y marzo de 2018.

Todos los pacientes con infecciones cervicofaciales graves fueron incluidos en el estudio. La infección cervicofacial grave se define como toda aquella que precisa de ingreso hospitalario para un adecuado manejo médico y/o quirúrgico.

Los criterios de ingreso hospitalario fueron: trismus (definido como una apertura oral menor de $40 \mathrm{~mm}$ ), disnea, disfagia, odinofagia, fiebre y malestar general.

Pacientes con infecciones cervicofaciales leves de cualquier origen, con buen estado general, drenaje espontáneo o posibilidad de drenaje bajo anestesia local, sin necesidad de ingreso, fueron excluidos del estudio.

Se analizaron múltiples variables entre las que se encuentran: etiología de la infección, sexo, clínica de presentación, aislamiento microbiológico, comorbilidades asociadas y los espacios cervicofaciales afectados. Además, se realizó una revisión del manejo terapéutico concretando si se llevó a cabo un tratamiento combinado médico-quirúrgico o únicamente médico, el tipo de antibioterapia, los días de hospitalización y las complicaciones observadas durante el ingreso.

El diagnóstico de la infección cervicofacial se llevó a cabo mediante una historia clínica detallada, una exploración física, una analítica incluyendo bioquímica y hemograma, junto con pruebas de imagen, una TC cervicofacial y una ortopantomografía.

Los datos analizados se presentan de forma descriptiva junto con una revisión de la literatura científica sobre el tema.

\section{RESULTADOS}

En la muestra analizada de 47 pacientes con infecciones cervicofaciales graves, 24 fueron mujeres (51\%) y 23 varones (49\%). La edad media de la muestra fue de 50,7 años, destacando que el $51 \%$ de la muestra eran pacientes menores de 50 años (Figura 1).

En cuanto a las comorbilidades asociadas, se encontró que 22 pacientes (47\%) no presentaban ninguna comorbilidad, mientras que el resto (53\%) sí presentaba alguna comorbilidad asociada o hábito tóxico, entre los que destacaron: alcohol y tabaco, hipertensión arterial y diabetes mellitus tipo 2 como las más frecuentes (Figura 2).
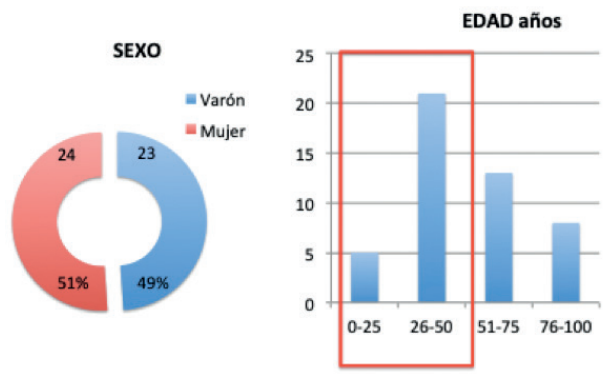

$=$ EDAD

Figura 1. Sexo y edad: alrededor de la mitad de los pacientes de la muestra eran varones y la otra mitad mujeres. El $51 \%$ de los pacientes eran menores de 50 años.

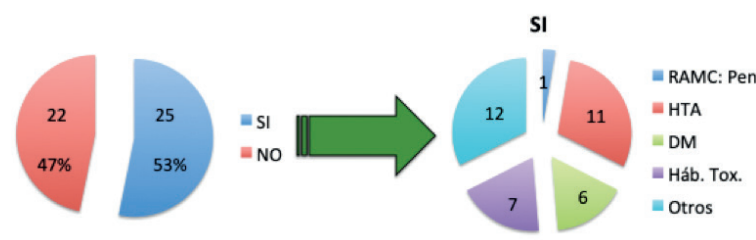

Figura 2. Comorbilidades: el $53 \%$ de los pacientes presentaron algún tipo de comorbilidad asociada. Entre las comorbilidades asociadas más frecuentes se encontraron los hábitos tóxicos (alcohol y tabaco), la hipertensión arterial y la diabetes mellitus.

Respecto a la etiología, la más frecuente fue la odontogénica en 43 pacientes (91\%) de los 47 incluidos en el estudio. En el resto se encontró como fuente principal de la infección cervicofacial: patología de glándulas salivales (4,25\%), adenoflemón (2,12 \%) y etiología no filiada (2,12\%).

Dentro del grupo de etiología odontogénica (43 pacientes), se apreció que en 8 pacientes la infección fue postexodoncia y en el resto (35 pacientes) fue por presencia de dientes en mal estado (caries y fractura de corona complicada). Además, se revisó qué piezas dentarias fueron las asociadas con mayor frecuencia a infecciones cervicofaciales graves. Encontramos que fueron los molares inferiores, en concreto los terceros molares inferiores, seguidos de primeros y segundos molares inferiores (Figura 3).

La clínica con la que se presentaron estos pacientes en el servicio de Urgencias con mayor frecuencia fue la tríada clínica de trismus, tumefacción facial y dolor. Hasta el 60 \% de los pacientes presentaron estos tres síntomas. Otros síntomas encontrados fueron: malestar general, fiebre, odinofagia, disfagia y rinolalia, entre otros.

De los 47 pacientes incluidos en el estudio, encontramos resultados de aislamiento microbiológico en 38 de ellos (80\%). En 9 pacientes no encontramos resultados microbiológicos. Si dividimos a los microrganismos aislados en aerobios y anaerobios, observamos que 20 fueron infecciones mixtas (aerobios

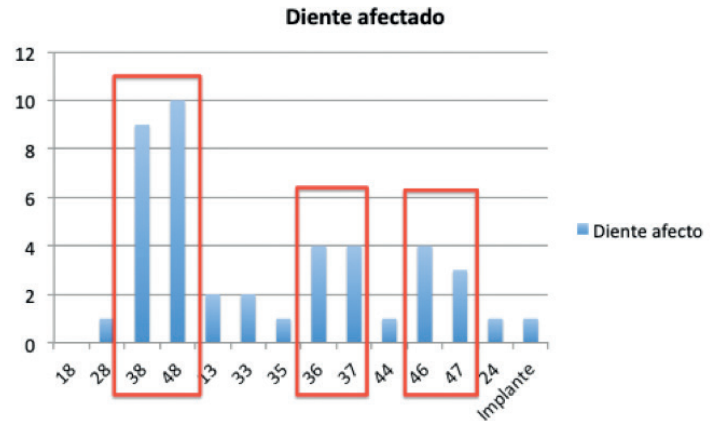

Figura 3. Etiología odontogénica: las piezas dentarias implicadas con mayor frecuencia en la etiología de infecciones cervicales graves en la muestra fueron los molares inferiores $y$, dentro de este grupo, los terceros molares. 
y anaerobios), en 10 se aislaron únicamente microrganismos aerobios, en 7 muestras se aislaron solo anaerobios y en otra muestra un hongo (Candida albicans). En el grupo de los microrganismos aerobios destaca el grupo de estreptococos (S. Constellatus y S. Epidermidis) y en el grupo de microrganismos anaerobios destaca Prevotella, Eikinella y enterobacterias (E. Cloacae y E. Coli) por orden de prevalencia.

El grupo de estreptococos fue el aislado con mayor frecuencia (30 muestras [78,9\%]). Dentro de este grupo, los más frecuentes fueron el Streptococcus Constellatus seguido del Streptococcus epidermidis. El grupo de Prevotella fue el segundo aislado en prevalencia $(26,31 \%)$ seguido de otros como Eikinella, enterobacterias, etc.

El espacio cervicofacial afectado con mayor frecuencia fue el espacio submandibular (24 pacientes, 55,8 \%), seguido del espacio sublingual (5 pacientes, 10,6\%). Otros espacios cervicofaciales afectados en menor frecuencia fueron: espacio canino, pterigo-mandibular, pterigo-maxilar, buccinador y parafaríngeo (Figura 4).

Casi la totalidad de los pacientes de la muestra recibió tratamiento combinado médico-quirúrgico, excepto un paciente que únicamente precisó tratamiento médico por drenaje espontáneo de material purulento a través de una fístula orocervical. El 63,8 \% de los pacientes ya estaba bajo tratamiento antibiótico ambulatorio previo a su llegada al Servicio de Urgencias, siendo la amoxicilina $500 \mathrm{mg}$ cada 8 horas el más administrado seguido de otros como espiramicina/metronidazol 1 comp. cada 6 horas o levofloxacino 500 mg cada 24 horas.

El tratamiento médico intrahospitalario consistió en sueroterapia, antibioterapia intravenosa empírica con amoxicilina-clavulánico $1 \mathrm{~g}$ cada 8 horas y corticoterapia intensiva con metilprednisolona $1 \mathrm{mg} / \mathrm{kg}$ peso cada 24 horas. En pacientes alérgicos a betaláctamicos se administró clindamicina 600 mg i.v. cada 8 horas, como tratamiento empírico de segunda línea.

El tratamiento quirúrgico se basó en el drenaje de material purulento acumulado en los espacios cervicofaciales afectados, retirada del foco causante de la infección (pieza dentaria lo más frecuente), toma de muestra para posterior análisis microbiológico junto con la colocación de drenajes intraorales u orocervicales. De todas las intervenciones quirúrgicas (46 pacientes), 17 (37\%) se realizaron bajo anestesia local, mientras que 29 (63\%) se realizaron bajo anestesia general.

En todos los pacientes que fueron intervenidos bajo anestesia local se colocaron drenajes tipo penrose intraorales, sin crear comunicación orocervical. De los pacientes intervenidos bajo anestesia general (29), en 15 de ellos se colocaron drenajes intraorales exclusivamente, mientras que en 14 de ellos el drenaje fue orocervical, comunicando cavidad oral con piel cervical o facial. Un paciente se presentó con un absceso odontogénico con diseminación cervical y mediastínica, originando una mediastinitis que precisó de un drenaje conjunto con cirugía torácica y con un ingreso prolongado de 45 días con resolución completa del cuadro clínico.

Se analizaron los días de ingreso de cada paciente, teniendo en cuenta si el paciente se había intervenido bajo anestesia local o anestesia general. La media de días de ingreso de la totalidad de la muestra, excluyendo al paciente que presentó una mediastinitis, fue de 4,9 días. Los pacientes intervenidos bajo anestesia general permanecieron ingresados de media 1 día más que aquellos que fueron intervenidos bajo anestesia local (Figura 5).

Por último, las complicaciones encontradas durante el ingreso fueron: un hematoma cervical postquirúrgico (complicación quirúrgica), dos pacientes se presentaron con un cuadro de obstrucción de vía aérea superior que precisaron traqueostomía previa a la intubación, dos pacientes necesitaron reintervención para nuevo drenaje de colección por una inadecuada evolución médica y un paciente desarrolló una mediastinitis secundaria a la infección cervicofacial.

\section{DISCUSIÓN}

De los 47 pacientes analizados, 24 eran varones (51\%) y 23 mujeres (49\%). Respecto al sexo, en otros estudios analizados, como el publicado por Shakya y cols. ${ }^{4}$ que analiza una muestra de 100 casos de infecciones odontogénicas, presentan un predominio del sexo masculino (71 \%) con una ratio varón:mujer de 2,5:1. Otro estudio retrospectivo publicado en el

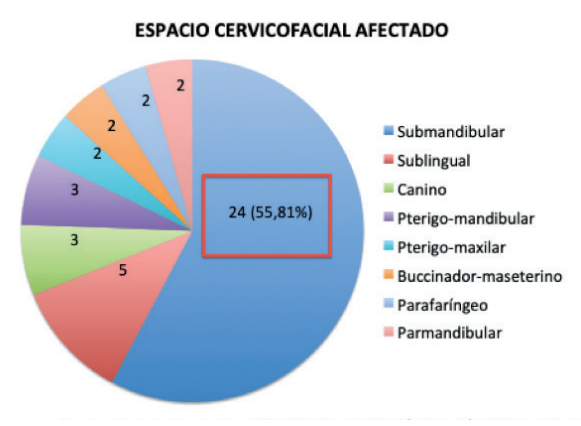

Figura 4. Espacio cervicofacial afectado: el espacio cervicofacial afectado con mayor frecuencia en la muestra a estudio fue el espacio submandibular (24 pacientes [55,8\%]), seguido del espacio sublingual (5 pacientes $[10,6 \%])$.

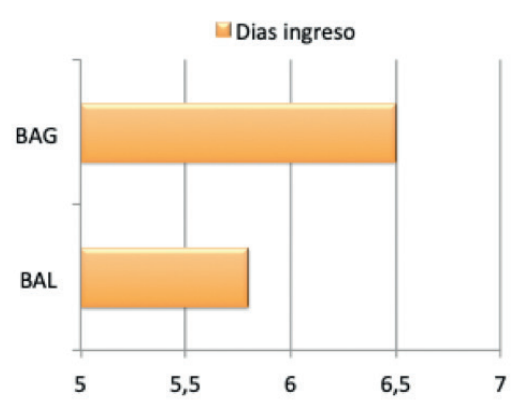

Figura 5. Duración del ingreso hospitalario: la duración media de ingreso de la totalidad de los pacientes de la muestra fue de 4,9 días. Los pacientes intervenidos bajo anestesia general (BAG) estaban ingresados de media un día más que los que fueron intervenidos bajo anestesia local (BAL). 
año 2016 por Mathew y cols. ${ }^{6}$, sobre una muestra de 136 pacientes con infecciones cervicofaciales odontogénicas, encuentra también una mayor afectación del hombre (66,4 \%).

La edad media fue de 50,77 años, encontrando hasta 24 pacientes (51\%) menores de 50 años. Los resultados encontrados nos muestran una mayor prevalencia de infecciones odontogénicas en el grupo de jóvenes menores de 50 años. Si comparamos estos resultados con otros artículos, como el publicado por Shakya y cols. ${ }^{4}$, publicado en el año 2017, observamos que son bastante similares aunque en este artículo la edad media obtenida era mucho menor que en el nuestro (37,35 años), siendo el grupo de edad más afectado por estas infecciones el situado entre los 20-40 años. Si analizamos otro artículo publicado en el año 2014 por Singh y cols. ${ }^{7}$, encontramos resultados similares: una edad media de 32,37 años y el grupo de edad más afectado fue el de 30-40 años.

Respecto a la etiología, en nuestro estudio ha destacado la odontogénica (91\%) como en la mayoría de la literatura publicada. Causas menos frecuentes de infecciones cervicofaciales son patología de glándulas salivares, adenitis cervicales, etc.

Dentro de la etiología odontogénica (43 de 47 pacientes), en 8 pacientes la infección apareció postexodoncia y en el resto por dientes en mal estado. En nuestro estudio destacaron los molares inferiores como las piezas dentarias causantes con mayor frecuencia de infecciones cervicofaciales graves y, dentro de este grupo, los terceros molares ${ }^{8,9}$. Revisando la literatura sobre qué piezas dentarias suelen ser las que con mayor frecuencia causan infecciones cervicofaciales graves, encontramos que en los estudios publicados por Moenning y cols. ${ }^{8}$ en 1989 o por Storoe y cols. ${ }^{9}$ en el año 2001 coinciden con los resultados de nuestro estudio, siendo los terceros molares inferiores los implicados con mayor frecuencia. Sin embargo, en el estudio publicado por Shakya y cols. ${ }^{4}$, publicado hace 2 años, los primeros molares inferiores fueron los causantes de mayor número de infecciones $(41,9 \%)$, seguidos de los segundos y terceros molares inferiores, $16,3 \%$ y $15,1 \%$, respectivamente.

El espacio cervicofacial afectado con mayor frecuencia en la muestra analizada fue el espacio submandibular (24 pacientes [51 \%]) seguido en segundo lugar por el espacio sublingual (5 pacientes). Estos resultados se correlacionan con otros estudios analizados en la literatura. Si revisamos el artículo publicado en el año 2012 por Mathew y cols. ${ }^{10}$, que consiste en un estudio restrospectivo que analiza 136 casos de infecciones cervicofaciales de origen odontogénico, obtiene que el espacio cervicofacial afectado con mayor frecuencia es el submandibular (69,3\%), similar a nuestro estudio, seguido de los espacios bucal, submental y sublingual. En otro estudio publicado en el año 2015 por el grupo de Opitz y cols. ${ }^{11}$, que analiza de forma retrospectiva hasta 814 pacientes con infección odontogénica grave en un periodo de 8 años, también se concluye que el espacio submadibular es el más afectado en su muestra con un 78,6 \% de los casos.

La clínica de presentación más frecuente en el Servicio de Urgencias de nuestro hospital fue la tríada clínica de tumefacción facial, trismus y dolor orofacial, estando presente esta tríada sintomática hasta en el $60 \%$ de los pacientes de la muestra (en 28 de 47 pacientes). Otros síntomas acompañantes fueron: disfagia, odinofagia, malestar general y fiebre entre otros.

Respecto a la sintomatología más frecuente encontrada en nuestro estudio coincide con otros estudios analizados de la lite- ratura, como el de Mathew y cols..$^{12}$, ya comentado previamente, en el que se encontró que los síntomas más prevalentes fueron tumefacción (100\%), seguido de dolor (97,1\%), trismus (50,4 \%) y fiebre.

Si comparamos los resultados microbiológicos obtenidos en nuestro estudio con los observados en la literatura actual encontramos que existe cierta similitud en cuanto a las especies aisladas con mayor frecuencia pero con variaciones considerables.

En nuestro estudio, de los 47 pacientes analizados pudimos obtener resultados microbiológicos de 38 pacientes (80\%), encontrando que la mayoría eran infecciones polimicrobianas. De los 38 cultivos, encontramos que 18 (47,3\%) eran infecciones mixtas (aerobios y anaerobios), 10 (26,31\%) infecciones por microrganismos aerobios, 7 (18,42\%) únicamente anaerobios y en una muestra solo se aisló una Candida Albicans. Si comparamos estos resultados con los obtenidos en el estudio de Singh y cols. ${ }^{7}$, publicado en 2014, que analizaron de forma retrospectiva 30 muestras de pacientes con infección cervicofacial de origen odontogénico, encontraron que el $43 \%$ de las muestras eran infecciones causadas por microorganismos anaerobios únicamente, el $39 \%$ causadas por aerobios y el $18 \%$ mixtas. Los datos aportados en este último estudio difieren claramente de los obtenidos del trabajo realizado en nuestro centro.

En cuanto a los microorganismos aerobios aislados con más frecuencia en nuestro estudio, sin lugar a duda fueron los estreptococos alfa hemolíticos coincidiendo con los resultados del estudio Singh y cols. ${ }^{7}$. Sin embargo, analizando las bacterias anaerobias, en nuestro estudio el género Prevotella es el más prevalente, mientras que en el estudio de Shing y cols. ${ }^{7}$ los anaerobios más frecuentes fueron los pertenecientes al grupo peptoestreptococos, seguido de bacteroides y prevotella.

Analizando el tratamiento realizado en nuestro centro, la totalidad de los pacientes recibieron tratamiento médico hospitalario (sueroterapia, corticoterapia y antibioterapia) y quirúrgico. El antibiótico empírico más utilizado fue la amoxicilina-clavulánico (95\%) seguido de la clindamicina en pacientes alérgicos a betalactámicos. Según los datos microbiológicos de múltiples estudios analizados, la penicilina sigue siendo el antibiótico de amplio espectro más administrado y con mejores resultados ${ }^{5,7,13}$.

Respecto al uso de los corticoides en el manejo de infecciones cervicofaciales graves persiste gran controversia en la literatura ${ }^{14}$ ya que, por un lado, por su efecto antinflamatorio e inmunomodulador mejoran sustancialmente la inflamación y resto de sintomatología asociada. Sin embargo, por otro lado, pueden alterar la respuesta fisiológica contra la infección y favorecer su diseminación.

En nuestro centro administramos corticoterapia intensiva intravenosa en los 4-5 primeros días de ingreso con metilprednisolona $1 \mathrm{mg} / \mathrm{kg} / 24$ horas, y si el paciente evoluciona favorablemente se suspende de forma gradual. Revisando la literatura actual sobre el tema destaca el estudio publicado en 2019 por Kent y cols. ${ }^{15}$, que consiste en una revisión sistemática sobre el papel de los corticoesteroides en el manejo de las infecciones de cabeza y cue1lo. En este estudio se concluye que la administración de cortico terapia a altas dosis y en un periodo corto de tiempo como medida adyuvante a la antibioterapia intravenosa y el manejo quirúrgico es una aproximación terapéutica efectiva y segura.

En cuanto a las complicaciones graves derivadas de la progresión de la enfermedad, se presentaron en un número relativamente bajo, teniendo en cuenta que en este estudio solo se 
han incluido pacientes con infecciones cervicofaciales graves $^{16}$. Un paciente se presentó con diseminación de la infección a mediastino, dos pacientes precisaron traqueostomía por dificultad en la intubación y complicaciones postquirúrgicas evolutivas, como un hematoma cervical, y necesidad de reintervenir a 3 pacientes para realizar nuevo drenaje por mala evolución clínica.

Respecto a la necesidad de traqueostomía en estas situaciones, el estudio publicado en el año 2017 por Tapiovaara y cols. ${ }^{17}$, en el que comparan los resultados obtenidos en pacientes con infecciones cervicales profundas que fueron intubados frente a aquellos en los que se realizó una traqueostomía, encontraron que en el grupo de pacientes que precisó traqueostomía el curso clínico fue más tórpido. Sin embargo, pacientes a los que se realizó traqueostomía permanecieron menos días de ingreso en UVI y menores complicaciones asociadas a intubación prolongada.

La mediastinitis cervical descendente consiste en una diseminación de una infección odontogénica u orofaríngea hacia mediastino a través de los espacios cervicales profundos. La infección odontogénica representa el 50 \% de la etiología de esta patología. Es una complicación muy poco frecuente y con una tasa de mortalidad entre el 20-50\%. En el estudio publicado en 2019 por Taylor y cols. ${ }^{17}$ concluyeron que un manejo quirúrgico lo más precoz posible en conjunto con cirujanos torácicos es esencial. Se pueden obtener unos resultados favorables si el manejo quirúrgico es precoz y agresivo.

\section{CONCLUSIONES}

En nuestra serie, la etiología más frecuente de infecciones cervicofaciales graves fue la odontogénica, debido a piezas dentarias en mal estado y, dentro de este grupo, los terceros molares fueron los dientes implicados con mayor frecuencia. La clínica de presentación característica fue tumefacción cervicofacial, trismus y dolor orofacial. El espacio cervicofacial afectado con mayor frecuencia fue el submandibular.

Las infecciones cervicofaciales graves se beneficiaron de un tratamiento combinado médico-quirúrgico. El antibiótico de amplio espectro más utilizado en nuestro centro fue la amoxicilina-clavulánico. Los pacientes en los que se precisó drenaje de colección purulenta bajo anestesia general estuvieron ingresados de media un día más que aquellos pacientes en los cuales el drenaje se realizó bajo anestesia local.

\section{B I B L I O G R A F Í A}

1. Byers J, Lowe T, Goodall CA. Acute cervico-facial infection in Scotland 2010: patterns of presentation, patient demographics and recording of systemic involvement. $\mathrm{Br} J$ Oral Maxillofac Surg. 2012;50(7):626-30. DOI: 10.1016/j.bjoms.2011.11.013.
2. Carter LM, Layton S. Cervicofacial infection of dental origin presenting to maxillofacial units in United Kingdom: a national audit. Br Dent J 2009;206(2):73-8. DOI: 10.1038/sj.bdj.2009.4.

3. Mc DonaldC, Hennedigeb A, Henryc A, Dawoudd B, Kulkarnie R, Gilbertf K, et al. Management of cervicofacial infections: a survey of current practice in maxillofacial units in the UK. Br J Oral Maxillofac Surg. 2017;55(9):940-5. DOI: 10.1016/j.bjoms.2017.09.002.

4. Shakya N, Sharma D, Newaskar V, Agrawal D, Shrivastava S, Yadav R. Epidemiology, Microbiology and Antibiotic Sensitivity of Odontogenic Space Infections in Central India. J Maxillofac Oral Surg. 2018;17(3):324-31. DOI: 10.1007/s12663-017-1014-y.

5. Rega AJ, Aziz SR, Ziccardi VB. Microbiology and Antibiotic Sensitivities of Head and Neck Space Infections of Odontogenic Origin. J Oral Maxillofac Surg. 2006;64(9):1377-80. DOI: 10.1016/j. joms.2006.05.023.

6. Mathew GC, Ranganathan LK, Gandhi S, Jacob ME, Singh I, Solanki M, et al. Odontogenic maxillofacial space infections at a tertiary care center in North India: a five-year retrospective study. Int J Infect Dis. 2012;16(4):296-302. DOI: 10.1016/j.ijid.2011.12.014.

7. Singh M, Kambalimath DH, Gupta KC. Management of odontogenic space infection with microbiology study. J Maxillofac Oral Surg. 2014;13(2):133-9. DOI: 10.1007/s12663-012-0463-6.

8. Moenning JE, Nelson CL, Kohler RB. The microbiology and chemotherapy of odontogenic infections. J Oral Maxillofac Surg 1989;47(9):976-85. DOI: 10.1016/0278-2391(89)90383-2.

9. Storoe W, Haug RH, Lillich TT. The changing face of odontogenic infections. J Oral Maxillofac Surg. 2001;59(7):739-48. DOI: 10.1053/ joms.2001.24285.

10. Poeschl PW, Spusta L, Russmueller G, Seemann R, Hirschl A, Poes$\mathrm{chl} \mathrm{E,} \mathrm{et} \mathrm{al.} \mathrm{Antibiotic} \mathrm{susceptibility} \mathrm{and} \mathrm{resistance} \mathrm{of} \mathrm{the} \mathrm{odon-}$ togenic microbiological spectrum and its clinical impact on severe deep space head and neck infections. Oral Surg Oral Med Oral Pathol Oral Radiol Endod. 2010;110(2):151-6. DOI: 10.1016/j. tripleo.2009.12.039.

11. Opitz D, Camerer C, Camerer DM, Raguse JD, Menneking H, Hoffmeister B, et al. Incidence and management of severe odontogenic infections-a retrospective analysis from 2004 to 2011. J Craniomaxillofac Surg. 2015;43(2):285-9. DOI: 10.1016/j. jcms.2014.12.002.

12. Rega AJ, Aziz SR, Ziccardi VB. Microbiology and antibiotic sensitivities of head and neck space infections of odontogenic origin. J Oral Maxillofac Surg. 2006;64(9):1377-80. DOI: 10.1016/j. joms.2006.05.023.

13. Ogle OE. Odontogenic Infections. Dent Clin N Am. 2017;61(2):23552. DOI: 10.1016/j.cden.2016.11.004.

14. Taub D, Yampolsky A, Diecidue R, Gold L. Controversies in the management of oral and maxillofacial infections. Oral Maxillofac Surg Clin North Am. 2017;29(4):465-73. DOI: 10.1016/j. coms.2017.06.004

15. Tapiovaara L, Bäck L, Aro K. Comparison of intubation and tracheotomy in patients with deep neck infection. Eur Arch Otorhinolaryngol. 2017;274(10):3767-72. DOI: 10.1007/ s00405-017-4694-5.

16. Osborn TM, Assael LA, Bell RB. Deep Space Neck Infection: Principles of Surgical Management. Oral Maxillofacial Surg Clin N Am. 2008;20(3):353-65. DOI: 10.1016/j.coms.2008.04.002.

17. Taylor M, Patel H, Khwaja S, Rammohan K. Descending cervical mediastinitis: the multidisciplinary surgical approach. European Archives of Oto-Rhino-Laryngology. 2019;276(7):2075-9. DOI: 10.1007/s00405-019-05471-z. 